S. Kwok, M. Dopita, and R. Sutherland, eds.

\title{
Formation of the 3-Ring Structure in the PN of the Progenitor of SN 1987A by an MHD Process
}

\author{
Takashi Tanaka \\ Communications Research Laboratory, Koganei-shi, Tokyo 184-8795, \\ Japan
}

Haruichi Washimi

Shonan Institute of Technology, Tsujido, Fujisawa 251-8511, Japan

\begin{abstract}
Optical observations by the HST have shown a fantastic 3ring structure in the circumstellar space of the SN 1987A. We attempt to reproduce the 3-ring structure from an MHD simulation which takes into account the wind-wind interaction between the slow, magnetized stellar wind generated while the progenitor was a red supergiant (RSG) and the subsequent fast stellar wind ejected from the same star after it had evolved into the blue supergiant (BSG). An important assumption in the simulation is that both the RSG and BSG winds have toroidal magnetic field and coronal holes. The simulation shows that the magnetic pinch effect is essential for the formation of the equatorial ring and that the amplification of initial density asymmetry by the effect of the dynamical interaction is responsible for the formation of two outer rings.
\end{abstract}

Recent optical observations by the Hubble Space Telescope (HST) show many ring-like structures of circumstellar nebulae that are associated with the death of stars. These structures are in contrast to the disk-like structures of interstellar gas associated with the birth of stars. One of the most beautiful and impressive of these ring-like structures is the 3-ring around the supernova (SN) 1987A (Panagia 1994). The central ring of the SN 1987A is on the equatorial plane of the star and has a diameter of 0.7 ly (light year). The two outer rings, at latitudes of about $45^{\circ}$ in the northern and southern hemispheres, have diameters about twice that of the equatorial ring (i.e., $1.4 \mathrm{ly}$ ). These three rings are the traces of high-density regions illuminated through the recombination process of atoms ionized by the UV flash of the central star. This paper clarifies the formation process of the 3-ring structure by computer simulation.

Theoretical discussions about the ring structures of planetary nebulae started with an idea of the hydrodynamic (HD) interaction between fast and slow stellar winds (Kwok 1982). However, if the gas is ionized and magnetized, the windwind interaction must be discussed in a magnetohydrodynamic (MHD) regime in which, in addition to the thermal pressure and the ram pressure (flow pressure), the magnetic pressure plays an important role for the ring formation. An MHD simulation of the single-ring formation was studied previously by Washimi 
et al. (1996) who assumed that the RSG wind has a toroidal magnetic field and a neutral sheet and that the density and velocity distributions are spherically symmetric around the star. After changing the inner boundary condition to that of the fast BSG wind, they obtained a localized high-density ring on the equatorial plane owing to the magnetic pinch effect.

We assume in this paper that the star has a dipole magnetic field along the rotation axis as is in our previous study, and in addition that the stellar wind has a coronal hole around the pole: the stellar wind is fast in the highlatitude coronal-hole region and slow in the low-latitude region. We indicate the boundary latitude between high- and low-speed regions by $\theta_{c}$. Our coronal-hole assumption is consistent with recent observations of the solar wind obtained by Ulysses (Phillips et al. 1995) in the polar orbit. Another important advantage of the present simulation is that it uses a highly accurate scheme for the MHD calculation. Our simulation adopts a finite-volume TVD scheme with a third-order numerical flux based on the monotonic upstream scheme for the conservation law (MUSCL) approach (Tanaka 1994).

The magnetic field near the star is carried out into the circumstellar space by the radially expanding stellar wind. Here we should notice that resulting radial component of the field, $B_{r}$ is not a monopole type, but is the so-called split-monopole type. That is, the direction of the radial field is outward (inward) in the northern hemisphere and inward (outward) in the southern hemisphere, following the polarity of the source dipole field. Thus a magnetic neutral sheet, where the field intensity is zero, is formed on the equatorial plane. In the circumstellar space of the star which is rotating with the rotational speed $\Omega$, the toroidal component of magnetic induction $B_{\phi}$ is formed as $B_{\phi}=\left(r \Omega / V_{r}\right) B_{r} \cos \theta$ , where $r$ is the distance from the star, $\theta$ the latitude, and $V_{r}$ the radial velocity of the stellar wind. Under the condition of the split-monopole of $B_{r}, B_{\phi}$ also reverses its polarity across the equatorial plane, and hence $B_{\phi}$ also forms the neutral sheet along the equator. In this paper the angular width of the equatorial neutral sheet is assumed to be $2 \delta \theta$. As a consequence, we find that the intensity of $B_{\phi}$ increases monotonically from the pole $\left(\theta=90^{\circ}\right)$ to near the equator $(\theta=\delta \theta)$ and then sharply decreases to zero just on the equator. Since $B_{r}$ decreases quickly with $r$ while $B_{\phi}$ does slowly, at a large distance from the star $B_{r}$ becomes negligible compared with $B_{\phi}$. Our simulation therefore ignores $B_{r}$ and considers only $B_{\phi}$.

We set the spherical inner and outer boundaries at $r=0.1 l y$ and at $r=$ $1.5 \mathrm{ly}$, respectively. We specify the inner boundary conditions for the RSG wind as follows: density $r=3 \cdot 10^{3} \times m_{p} g / \mathrm{cm}^{3}$ ( $m_{p}$ :proton mass), temperature $T=5 \times 10^{3} \mathrm{~K}, V_{r}=10 \mathrm{~km} / \mathrm{s}$, and $B_{\theta}=B_{0} \cos \theta$ with $B_{0}=37 n T$. Assuming high-speed hot plasma in the coronal hole, we modify these primary boundary conditions at $|\theta|>\theta_{c}$ by increasing $V_{r}$ by a factor of 4.0 and increasing $T$ by a factor of 2.0. Despite these changes, the fluxes $\rho V_{r}$ and $B_{\phi} V_{r}$ are held constant at all latitudes ( $\rho$ and $B_{\phi}$ are decreased by a factor of $1 / 4$ at $|\theta|>\theta_{c}$ ). For the RSG, $\theta_{c}$ is set at $55.7^{\circ}$ and $\delta \theta$ is set to be $1.5^{\circ}$. The boundary conditions for the BSG are specified as $\rho=160 \times m_{p} g / \mathrm{cm}^{3}, T=4 \times 10^{4} \mathrm{~K}, V_{r}=460 \mathrm{~km} / \mathrm{s}$, and $B_{0}=6.6 n T$; and $\theta_{c}$ for the BSG is set at $21.1^{\circ}$. At $|\theta|>\theta_{c}$, the rate of $V_{r}$ increase is changed from 3.0 at $\theta= \pm \theta_{c}$ to 4.0 at $\theta= \pm 90^{\circ}$. At $|\theta|<\theta_{c}$, 


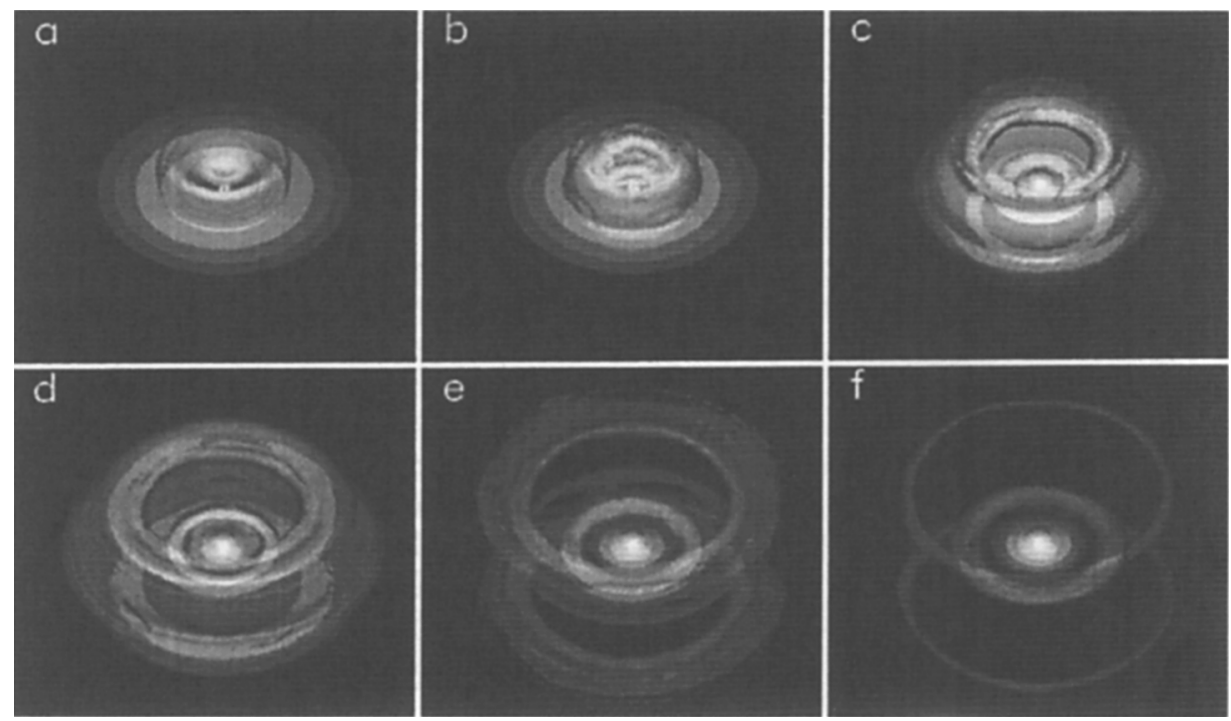

Figure 1. Time sequence of simulated plasma-density distribution around the SN 1987A. Thee-dimensional isodensity surfaces are shown at four number levels $(0.12,0.06,0.04$, and 0.03$) \times 3 \cdot 10^{3} \mathrm{~cm}^{-3}$ for a, an initial state when the star was a red supergiant (RSG), and b-f, successive developments after the star had changed to a blue supergiant (BSG). White spheres show the position of the central star.

$V_{r}$ is reduced by 0.5 assuming that the flow is decelerated before reaching the inner boundary due to the relatively dense plasma around the equator.

Figure 1a shows the three-dimensional structure of the steady RSG wind obtained after a sufficient time of numerical integration under a fixed set of RSG inner boundary conditions. Three-dimensional isodensity surfaces are shown here at four density levels. In Figure 1a the density basically falls with $\mathrm{r}$ as $r^{-2}$ because of the radial expansion. The disk-like structure shows dense neutral sheet plasma in the equatorial plane. This density enhancement is due to the equatorward magnetic-pressure force (pinch effect) of $B_{\phi}$. The density is relatively low in the high-latitude coronal hole where the wind speed is high. In addition, broad density enhancement occurs on the low-latitude sides of the boundary at $\theta= \pm \theta_{c}$. This density increase, though not as clearly visible as the equatorial disk, is due to the poleward compression of plasma by the magnetic pressure at $|\theta|<\theta_{c}$ and the high ram pressure at $|\theta|>\theta_{c}$, which blocks the poleward pressure at $\theta= \pm \theta_{c}$. Figures $1 \mathrm{~b}$-f show the evolution of the density distribution in the circumstellar space after the BSG wind is turned on at the inner boundary. In Figures 1b-f the BSG wind is almost invisible because its density is low. The evolution of the density distribution shown here is, therefore, generated by the redistribution of RSG plasma in the course of the wind-wind interaction. The high-speed flow induced by the BSG wind gathers the RSG plasma in the circumstellar space in front of the contact surface. Then this surface of dense RSG plasma is inflated like a balloon (Figures 1c-d). At this stage, 
RSG plasma in the polar region is blown off by the fast BSG flow. In the course of the inflation, local concentrations of RSG plasma occur in three latitudinal regions: one at the equator corresponding to the equatorial ring, and two at the northern and southern midlatitudes corresponding to the outer rings. Near the equator, $B_{\phi}$ originally distributed in the RSG wind and gathered in front of the contact surface exerts a magnetic pinch effect to accelerate the equatorial-ring formation. In the outer rings, however, both the magnetic intensity and plasma density become greater than those of the initial state because both components are compressed simultaneously under the frozen-in condition. Hence, the outer rings are confined not by the surrounding magnetic pressure, but by the ram pressure built up in the BSG wind so as to surround the outer rings. In Figure 1c, the BSG wind is decelerated more severely around high-density ring areas. This deceleration in turn generates high-pressure walls around the outer rings and spurs the ring formation. Finally, the fast wind blows off RSG plasma in the crevices of the rings and makes the three rings stand out more clearly (Figures 1e-f). Figure $1 \mathrm{f}$ shows the three-dimensional isodensity surfaces at the time stage corresponding to Figure 1. Comparing the HST image (Panagia 1994) with Fig. 1f, we find that our simulation results and the HST observations correspond fairly well. At the final stage (Figure 1f), the radial velocity of the outer rings is $1 / 8$ of that of the surrounding BSG wind, and the density contrast between the outer rings and surrounding plasma is 30 on the front side, and 3 on the back side. Further work will be required for more accurate discussions about absolute values.

It is worth noticing that the final shape of the ring structure depends on the parameters of the RSG and BSG, such as $\theta_{c}$ and $B_{\phi}$, indicating that it may be possible to study the structures of the RSG or BSG in other SNs by finding simulation parameters that generate the rings actually observed. Our simulation suggests that the structures of the RSG and BSG exhibit many similarities to those of the sun. The formation process of the outer rings indicates that both the RSG and BSG winds should have high-speed polar streams as the solar wind does. Moreover they should also have magnetic fields as the sun does. In the RSG wind, the intensity of the magnetic pressure should be on the same order of magnitude as that of the thermal pressure. It is striking to note, however, that the present results suggest that the RSG wind should be blowing fairly steadily for a long period for the 3-ring formation, in contrast to that the solar wind is always undergoing long- and short-period variations.

Acknowledgments. We would like to thank Dr. S. Shibata for valuable discussions.

\section{References}

Kwok, S. 1982 Astrophys. J. 258, 280

Panagia, N. 1994 Nature 369, 354

Tanaka, T. 1994 J. Comput. Phys. 111, 381

Washimi, H., Shibata, S. \& Mori, M. 1996 Publ. Astron. Soc. Japan 48, 2

Phillips, J. L. et al. 1995 Geophys. Res. Lett. 22, 3301 